\title{
CONTRIBUTOS DA ALIMENTAÇÃO SAUDÁVEL COMO ESTRATÉGIA DE PREVENÇÃO E ENFRENTAMENTO DO CÂNCER: UMA PERSPECTIVA DA ENFERMAGEM
}

\section{CONTRIBUTIONS OF HEALTHY EATING AS A STRATEGY FOR PREVENTING AND COPING WITH CANCER: A PERSPECTIVE FROM NURSING}

Amanda de Araujo Franco ${ }^{1}$, Amanda de Almeida Floriano ${ }^{2}$, Ana Beatriz Teodoro de Souza ${ }^{3}$, Bianca Lemos de Carvalho ${ }^{4}$, Jully Camara Guinancio ${ }^{5}$, Júlio Gabriel Mendonça de Sousa ${ }^{6}$, Wanderson Alves Ribeiro ${ }^{7}$

Submetido em: 19/04/2021

e24214

Aprovado em: 30/04/2021

\section{RESUMO}

O câncer consiste em uma enfermidade crônica, caracterizada pelo crescimento desordenado das células, o qual é resultante de alterações no código genético. Através de estudos epidemiológicos, associações relevantes podem ser feitas entre alguns padrões alimentares observados em diferentes regiões do mundo e a prevalência de câncer. Trata-se de uma pesquisa de revisão bibliográfica da literatura, de abordagem qualitativa e caráter descritivo. A amostra final foi composta apenas por 9 referências, incluindo apenas as de maior relevância para este estudo. Posterior à leitura reflexiva emergiram três categorias: Principais contribuições da modificação na alimentação para prevenção do câncer; Importância da alimentação saudável para o paciente oncológico e Estratégias de enfrentamento frente a necessidade de melhora em qualidade de vida no âmbito nutricional na perspectiva da enfermagem. Concluindo-se que para promover orientações sobre alimentação saudável e nutrição adequada é preciso estar atento ao contexto social cujos indivíduos estão inseridos, abordando estratégias individualizadas em concordância com a necessidade exposta para uma eficaz manutenção da saúde pública.

PALAVRAS-CHAVE: Enfermagem. Alimentação Saudável. Câncer. Hábitos Saudáveis.

\begin{abstract}
Cancer consists of a chronic disease, characterized by the disordered growth of cells, which is the result of changes in the genetic code. Through epidemiological studies, relevant associations can be made between some dietary patterns observed in different regions of the world and the prevalence of cancer. This is a literature review survey of the literature, with a qualitative approach and descriptive character. The final sample consisted of only 9 references, including only the highest category for this study. After the reflective reading, three categories emerged: Main contributions of dietary modification for cancer prevention; Importance of healthy eating for cancer patients and Coping strategies in view of the need to improve quality of life in the nutritional field from the perspective of nursing. In conclusion, in order to promote guidelines on healthy and adequate food, it is necessary to be attentive to the public context belonging to them, inserted, addressing individualized ones in accordance with the need to be exposed for an effective maintenance of health.
\end{abstract}

KEYWORDS: Nursing. Healthy eating. Cancer. Healthy habits.

\footnotetext{
${ }^{1}$ Enfermeira. Graduada pela Universidade Iguaçu, Nova Iguaçu, RJ, Brasil

${ }^{2}$ Enfermeira. Graduada pela Universidade Iguaçu, Nova Iguaçu, RJ, Brasil

3 Enfermeira. Graduada pela Universidade Iguaçu, Nova Iguaçu, RJ, Brasil

${ }^{4}$ Enfermeira. Graduada pela Universidade Iguaçu, Nova Iguaçu, RJ, Brasil

${ }^{5}$ Enfermeira. Graduada pela Universidade Iguaçu, Nova Iguaçu, RJ, Brasil

${ }_{7}^{6}$ Acadêmico da Graduação de Enfermagem da Universidade Federal do Rio de Janeiro. RJ, Brasil.

7 Enfermeiro. Mestre e Doutorando pelo programa acadêmico em ciências cuidado em saúde da EEAAC/UFF; Docente do curso de graduação em enfermagem da Universidade Iguaçu.
} 


\section{RECIMA21 - REVISTA CIENTÍFICA MULTIDISCIPLINAR ISSN 2675-6218}

CONTRIBUTOS DA ALIMENTAÇÃO SAÚDAVEL COMO ESTRATÉGIA DE PREVENÇÃO E ENFRENTAMENTO DO CÂNCER: UM PERSPECITIVA DA ENFERMAGEM Amanda de Araujo Franco, Amanda de Almeida Floriano, Ana Beatriz Teodoro de Souza, Bianca Lemos de Carvalho, Jully Camara Guinancio, Júlio Gabriel Mendonça de Sousa, Wanderson Alves Ribeiro

\section{INTRODUÇÃO}

O câncer consiste em uma enfermidade crônica, caracterizada pelo crescimento desordenado das células, o qual é resultante de alterações no código genético. De 5\% a 10\% das neoplasias são resultados diretos da herança de genes relacionados ao câncer e grande parte dos casos envolve danos ao material genético, de origem física, química ou biológica que se acumulam ao longo da vida. ${ }^{1}$

O desenvolvimento de várias das formas mais comuns de câncer resulta de uma interação entre fatores modificáveis e não modificáveis, sendo o mais aparente desses fatores a dieta e o estilo de vida. Segundo Oliveira et al. ${ }^{2}$, através de estudos epidemiológicos, associações relevantes podem ser feitas entre alguns padrões alimentares observados em diferentes regiões do mundo e a prevalência de câncer.

Outros fatores ambientais, tais como o tabagismo, a obesidade, a atividade física e a exposição a tipos específicos de vírus, bactérias e parasitas, além do contato frequente com algumas substâncias carcinogênicas como produtos de carvão e amianto, agrotóxicos também merecem ser observados ${ }^{3}$. Segundo a Organização Mundial de Saúde (OMS), O câncer é a segunda principal causa de morte no mundo e é responsável por 9,6 milhões de mortes em 2018. A nível global, uma em cada seis mortes são relacionadas à doença. ${ }^{4}$

Gurgel $^{5}$ afirma que no Brasil, tal doença representa a segunda causa de morte para ambos os sexos, sendo superado apenas pelas doenças cardiovasculares. Estudos epidemiológicos demonstram estreita relação entre câncer, obesidade, estilo de vida e nutrição. Contudo, verifica-se que modificações na alimentação e no estilo de vida podem prevenir alguns tipos dessa doença. ${ }^{6}$ Como medida de prevenção para o câncer, diversos artigos evidenciam a importância da introdução de alimentos na dieta que contenham propriedades antioxidantes, sendo preconizado o alto consumo de frutas e hortaliças. ${ }^{7}$

Nenhum alimento por si só é capaz de proteger contra o câncer, entretanto, a combinação correta de determinados alimentos pode estimular o sistema imunológico a lutar contra a doença. Quando se trata de câncer, porém, a alimentação tem valor efeito preventiva, entretanto, quando já diagnosticada a doença a dieta continua a representar importante papel no tratamento. ${ }^{8}$

Fundamentando-se em estudos epidemiológicos, é evidente a relação existente entre câncer e nutrição, além do poder que a alimentação possui por combater a origem e o desenvolvimento de tumores, por meio de escolhas alimentares saudáveis. Alguns compostos denominados agentes quimiopreventivos, exercem uma ação protetora específica contra o desenvolvimento do câncer, muitos desses compostos químicos podem ser sintetizados em laboratórios, entretanto, a maioria deles encontram-se diretamente disponível nos alimentos. ${ }^{9}$ 


\section{RECIMA21 - REVISTA CIENTÍFICA MULTIDISCIPLINAR ISSN 2675-6218}

CONTRIBUTOS DA ALIMENTAÇÃO SAÚDAVEL COMO ESTRATÉGIA DE PREVENÇÃO E ENFRENTAMENTO DO CÂNCER: UM PERSPECITIVA DA ENFERMAGEM Amanda de Araujo Franco, Amanda de Almeida Floriano, Ana Beatriz Teodoro de Souza, Bianca Lemos de Carvalho, Jully Camara Guinancio, Júlio Gabriel Mendonça de Sousa, Wanderson Alves Ribeiro

A alimentação adequada, variada e sem exageros, pode contribuir para uma saúde ideal e equilibrada. A ingestão adequada e regular desses alimentos pode contribuir para a redução de 5-12\% dos casos de câncer, além de serem ricos em vitaminas e minerais que atuam fortalecendo o sistema imune. A OMS recomenda um consumo diário de pelo menos cinco porções de frutas, legumes e verduras em torno de $400 \mathrm{~g} / \mathrm{dia}$. O consumo regular de grandes quantidades de frutas e vegetais, incluindo alimentos à base de soja, ajuda a prevenir alguns tipos de câncer. ${ }^{10}$

Publicações abalizadas por instituições como a OMS, a organização das nações unidas para a alimentação e a agricultura (FAO) e o Fundo Mundial para Pesquisa em câncer (WCRF) apontam que os fatores mais importantes para o aumento do excesso de peso corporal, assim como de outras doenças crônicas não transmissíveis relacionadas, são a prática insuficiente de atividade física e o elevado consumo de alimentos e preparações com alto teor de gordura Baseado nos critérios do Modelo de Perfil Nutricional da Organização Pan-Americana da Saúde. ${ }^{11}$

Em 2015, a International Agency for Research on Cancer (IARC), agência de pesquisa sobre câncer da OMS, classificou o consumo de carne vermelha como provável agente carcinogênico para os seres humanos. Além disso, a carne processada é classificada como evidentemente cancerígena para os seres humanos, baseando-se em comprovações que correlacionem seu consumo ao desenvolvimento de câncer colorretal (CCR), posto que o consumo diário de uma porção de 50 gramas de carne processada aumenta $18 \%$ o risco de manifestação dessa neoplasia. ${ }^{12}$

A IARC classifica os compostos em quatro grupos com base nas evidências científicas disponíveis para aumento do risco de câncer em animais e seres humanos. As quatro classificações são os Grupos 1, 2A, 2B, 3 e 4, que correspondem à diminuição das evidências disponíveis para o risco de câncer. ${ }^{12}$

Em face do exposto é evidente que uma alimentação equilibrada em quantidade e qualidade, composta por macronutrientes e rica em fibras, vitaminas e minerais atua na promoção da saúde e bem-estar como um todo, bem como para a prevenção do câncer. Nesse sentido, o déficit de informação sobre como equilibrar e adequar a alimentação pode causar impactos na vida das pessoas.

Desta forma, estabeleceu-se nesta pesquisa como objetivo descrever as principais contribuições da alimentação saudável, como fator modificável, para prevenção do câncer; refletir sobre a importância da alimentação saudável para o paciente oncológico e ainda, correlacionar estratégias de enfrentamento frente a necessidade de melhora em qualidade de vida no âmbito nutricional na perspectiva da enfermagem.

\section{METODOLOGIA}

RECIMA21 - Ciências Exatas e da Terra, Sociais, da Saúde, Humanas e Engenharia/Tecnologia 


\section{RECIMA21 - REVISTA CIENTÍFICA MULTIDISCIPLINAR ISSN 2675-6218}

CONTRIBUTOS DA ALIMENTAÇÃO SAÚDAVEL COMO ESTRATÉGIA DE PREVENÇÃO E ENFRENTAMENTO DO CÂNCER: UM PERSPECITIVA DA ENFERMAGEM Amanda de Araujo Franco, Amanda de Almeida Floriano, Ana Beatriz Teodoro de Souza, Bianca Lemos de Carvalho, Jully Camara Guinancio, Júlio Gabriel Mendonça de Sousa, Wanderson Alves Ribeiro

Trata-se de uma pesquisa de revisão bibliográfica da literatura, de abordagem qualitativa e caráter descritivo. Cabe ressaltar que a pesquisa bibliográfica que é desenvolvida com auxílio de material já elaborado, constituído principalmente de livros e artigos científicos. Contudo em grande parte dos estudos seja exigido algum tipo de trabalho deste gênero, há pesquisas desenvolvidas exclusivamente a partir de fontes bibliográficas. ${ }^{13}$

Segundo Lakatos e Marcone, ${ }^{14}$ conhecimento científico determina a utilização de métodos científicos; por outro lado, não são todos os estudos que utilizam esse modelo, reconhecido como ciência.

Perante a certificação, pode-se deduzir que a aplicação de métodos científicos não é competência específica da ciência, com tudo não existe ciência sem o uso de métodos científicos. Como tal característica, o método é a agregação de atividades sistemáticas e lógicas que, permite com total segurança e economia, atingir o objetivo, com estudos validos e verdadeiros, elaborando roteiros a seres seguidos, encontrando erros e contribuindo com soluções dos cientistas. ${ }^{14}$

$\mathrm{Na}$ atualidade têm-se uma farta e complexa quantidade de dados na área da saúde, fazendo assim, com que haja necessidade de desenvolvimento de artigos e pesquisas, com embasamento científico, para possibilitar melhor delimitação metodológica esclarecendo diversos estudos. Mediante a necessidade, utilizamos a revisão bibliográfica como uma forma de metodologia que possibilita um apanhado de conhecimentos e aplica-se em resultados de estudos concisos na prático do profissional. ${ }^{13}$

Abordagem qualitativa é aquela que não trabalha com informações numéricas, mas sim, que trabalha com conceitos, ideologias, processos de comunicação humana, entre outros. E apresenta facilidade de definir hipótese ou problema, de explorar a interação de certas variáveis, de compreender e classificar processos dinâmicos experimentados por grupos sociais, de apresentar mudanças, elaboração ou formação de posição de determinados grupos, e de permitir, em grau de profundidade, a interpretação dos comportamentos ou atitudes dos indivíduos. ${ }^{13}$

Foram realizadas buscas na base de dados da Biblioteca Virtual em Saúde (BVS) Bireme, em janeiro de 2021, nas bases de dados: Literatura Lático Americana e do Caribe em Ciência da Saúde (LILACS), Bases de Dados da Enfermagem (BDENF), Biblioteca Eletrônica Cientifica Online (SciELO).

Para a busca das referências foram utilizados os descritores "Enfermagem"; "Alimentação saudável"; "Hábitos saudáveis" advindos do sistema de Descritores em ciências da saúde (DeCS), utilizando o marcador “AND”. Para resgaste dos artigos, consideramos como critérios para inclusão de artigos publicados no período compreendido no mês de janeiro de 2021 com textos completos em língua portuguesa. E os critérios de exclusão foram os artigos repetidos, publicações com textos não disponíveis, fora da língua vernácula e estudos publicados em 2020 sobre a alimentação sem aderência a enfermagem. 


\section{RECIMA21 - REVISTA CIENTÍFICA MULTIDISCIPLINAR ISSN 2675-6218}

CONTRIBUTOS DA ALIMENTAÇÃO SAÚDAVEL COMO ESTRATÉGIA DE PREVENÇÃO E ENFRENTAMENTO DO CÂNCER: UM PERSPECITIVA DA ENFERMAGEM Amanda de Araujo Franco, Amanda de Almeida Floriano, Ana Beatriz Teodoro de Souza, Bianca Lemos de Carvalho, Jully Camara Guinancio, Júlio Gabriel Mendonça de Sousa, Wanderson Alves Ribeiro

Cabe mencionar que os textos em língua estrangeira foram excluídos devido ao interesse em embasar o estudo com dados do panorama brasileiro e os textos incompletos, para oferecer melhor compreensão através da leitura de textos na integra.

\section{RESULTADOS E DISCUSSÃO}

Optou-se pela busca com os descritores associados em trio, visando os encontros dos artigos de forma mais objetiva, respeitando a temática da construção teórica. Os resultados dessa busca se encontram descritos na Figura 1.

Figura 1 - Fluxograma da distribuição quantitativa das produções científicas encontradas nas bases de dados com os descritores associados em trio.

\section{Enfermagem $A N D$ \\ Alimentação \\ saudável $A N D$ \\ Hábitos saudáveis}
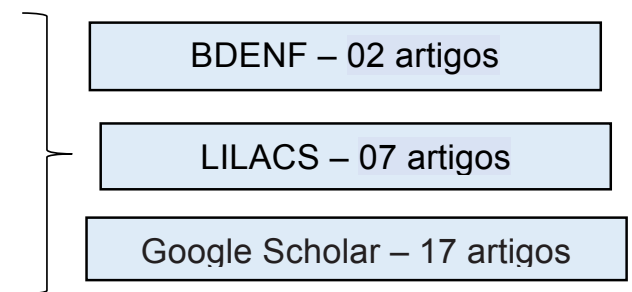

Google Scholar - 17 artigos
Processo de revisão das referências
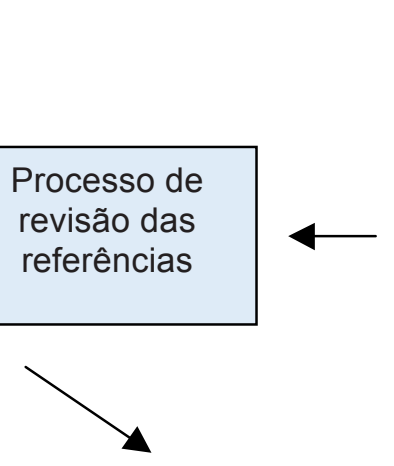

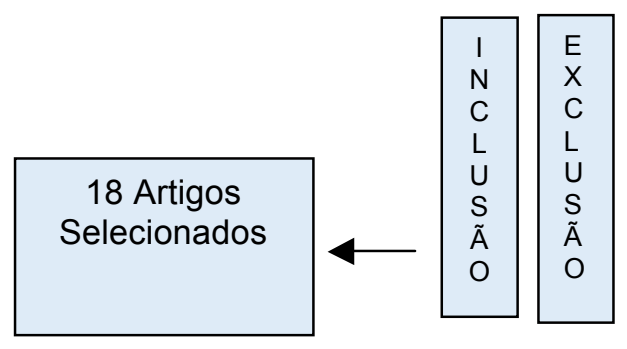

26 Artigos Encontrados

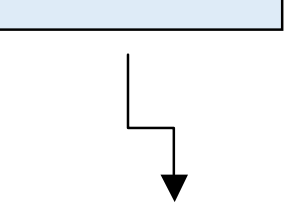

Critérios de Seleção dos Artigos

\section{Artigos Selecionados}

Fonte: Produção dos autores, 2021.

Finalizado esse percurso de busca, realizou-se aplicabilidade dos critérios de inclusão e exclusão apresentados acima e ainda, leitura dos resumos e os que apresentavam relevância para subsidiar a discussão do tema foram selecionados e lidos na íntegra.

Posterior à leitura reflexiva emergiram três categorias: Principais contribuições da modificação na alimentação para prevenção do câncer; Importância da alimentação saudável para o paciente oncológico e Estratégias de enfrentamento frente a necessidade de melhora em qualidade de vida no âmbito nutricional na perspectiva da enfermagem. 
RECIMA21 - REVISTA CIENTÍFICA MULTIDISCIPLINAR ISSN 2675-6218

CONTRIBUTOS DA ALIMENTAÇÃO SAÚDAVEL COMO ESTRATÉGIA DE PREVENÇÃO E ENFRENTAMENTO DO CÂNCER: UM PERSPECITIVA DA ENFERMAGEM Amanda de Araujo Franco, Amanda de Almeida Floriano, Ana Beatriz Teodoro de Souza, Bianca Lemos de Carvalho, Jully Camara Guinancio, Júlio Gabriel Mendonça de Sousa, Wanderson Alves Ribeiro

Uma vez criadas às categorias de análise, partiu-se para a fase final de inferência e discussão dos dados obtidos, mediante o respaldo obtido através da articulação entre o conteúdo verificado nas produções científicas e a atitude crítico-reflexiva dos pesquisadores.

\section{CATEGORIA 1 - PRINCIPAIS CONTRIBUIÇÕES DA MODIFICAÇÃO NA ALIMENTAÇÃO PARA PREVENÇÃO DO CÂNCER}

Estudos apontam que a redução dos riscos para o desenvolvimento do câncer, depende de uma interação entre fatores intrínsecos e extrínsecos, principalmente a nutrição e o estilo de vida adotado por cada indivíduo, influenciando diretamente em sua qualidade de vida, podendo ser contribuinte na prevenção ao câncer ou como um meio prejudicial à saúde, possibilitando o desencadeamento de células cancerígenas. ${ }^{[16]}$

Ainda segundo Munhoz et al. ${ }^{16}$, diante do exposto acima, entende-se que os padrões alimentares, a inatividade física, a obesidade, o tabagismo e a exposição frequente à agentes cancerígenos, tem relação com a prevalência do câncer, e a associação desses multifatores, contribuem fortemente para o desequilíbrio do organismo, tendo capacidade de ocasionar efeitos nocivos à saúde e potencializar o desenvolvimento de tumores no organismo.

Sabe-se que os hábitos alimentares têm papel fundamental na saúde do indivíduo, costumes estabelecidos desde a infância que podem refletir por toda a vida, e ter repercussões negativas, como o desencadeamento de tumores. ${ }^{17}$ No entanto, o consumo de alimentos ricos em gorduras, açúcares, conservantes e alimentos processados, vem aumentando em todo mundo, e o enfermeiro tem um papel indispensável no incentivo à manutenção do estilo de vida dos indivíduos e na adesão a hábitos mais saudáveis, reduzindo a probabilidade de implantação de um quadro oncológico. ${ }^{18}$

Sendo assim, é possível observar diversos artigos que evidenciam a importância da adoção à uma alimentação equilibrada, reduzindo o consumo frequente de carnes vermelhas e gorduras saturadas, e introduzindo uma dieta que envolva propriedades antioxidantes, enfatizando o consumo de fibras, que facilitam a remoção dos agentes carcinogênicos, e de frutas, verduras e legumes, pois auxiliam na promoção da saúde de modo em geral, como também, na prevenção ao câncer e no fortalecimento do sistema imunológico. No âmbito em questão, é pertinente salientar que a forma de preparo dos alimentos e as substâncias ali presentes, também influenciam em seu potencial cancerígeno. ${ }^{19}$

Nascimento, diz que nesse contexto, a associação de um acompanhamento nutricional dentro da rotina alimentar, estabelece um maior cuidado com a saúde e com repercussões mais efetivas, mediante a um olhar profissional, de forma individual, específica e de acordo com as necessidades de cada indivíduo, onde o profissional irá observar todo o cenário nutricional, e direcioná-lo para o melhor caminho, com orientações para o entendimento e 


\section{RECIMA21 - REVISTA CIENTÍFICA MULTIDISCIPLINAR ISSN 2675-6218}

CONTRIBUTOS DA ALIMENTAÇÃO SAÚDAVEL COMO ESTRATÉGIA DE PREVENÇÃO E ENFRENTAMENTO DO CÂNCER: UM PERSPECITIVA DA ENFERMAGEM Amanda de Araujo Franco, Amanda de Almeida Floriano, Ana Beatriz Teodoro de Souza, Bianca Lemos de Carvalho, Jully Camara Guinancio, Júlio Gabriel Mendonça de Sousa, Wanderson Alves Ribeiro

aceitação às modificações necessárias, com introdução de refeições mais saudáveis e balanceadas em seu dia a dia. ${ }^{20}$

O autor ${ }^{20}$ ainda acrescenta que, a implementação de condições mais favoráveis é uma estratégia admissível para promover uma melhor adaptação às mudanças de hábitos alimentares, viabilizando os benefícios acarretados pela alteração no padrão nutritivo, evidenciando uma melhoria da qualidade de vida e, consequentemente, reduzindo os riscos relacionados aos diversos tipos de câncer.

\section{CATEGORIA 2 - IMPORTÂNCIA DA ALIMENTAÇÃO SAUDÁVEL PARA O PACIENTE ONCOLÓGICO}

Dieta e câncer dispõem de uma ligação muito complexa, por isso muitas questões ainda não possuem respostas; no entanto, sabe-se que diversos alimentos contêm agentes carcinogênicos possibilitando o aumento da incidência do câncer. Certas substâncias alimentares podem alterar a estrutura química das células, interferindo no surgimento e no prognóstico da doença. ${ }^{21}$ Podemos citar como indutores do crescimento exacerbado de células tumorais as gorduras saturadas e hidrogenadas, bem como os corantes artificiais e conservantes, com ênfase nos alimentos industrializados. ${ }^{22}$

Pacientes com tumores malignos realizam o tratamento por meio de radiação ionizante, denominado de Radioterapia. A capacidade de proliferação ilimitada é uma característica bastante comum entre as células malignas, a velocidade na qual se multiplicam pode variar de acordo com o tipo de tumor. A radiação ionizante interfere na divisão celular, ao interagir com os tecidos, dá origem a elétrons rápidos que ionizam o meio e criam efeitos químicos como a hidrólise da água e a ruptura das cadeias de DNA. A morte celular pode ocorrer então por variados mecanismos, desde a inativação de sistemas vitais para a célula até sua incapacidade de reprodução. Entretanto, a morte celular pode não ocorrer imediatamente, mas ser retardada por várias divisões celulares que se prolongam de horas a meses. ${ }^{23}$ A quimioterapia é o método que utiliza compostos químicos, chamados quimioterápicos, no tratamento de doenças causadas por agentes biológicos. Quando aplicada ao câncer, a quimioterapia é chamada de quimioterapia antineoplásica ou quimioterapia antiblástica e pode ou não estar associada à radioterapia. ${ }^{24,20}$

Assim como a radioterapia, a quimioterapia possui grande impacto sobre a divisão das células tumorais e provoca toxicidade pelo efeito deletério sobre a divisão das células normais do corpo tais como a medula óssea, pelos e trato gastrintestinal. ${ }^{25,24,26}$

Tais tratamentos geram irritação da mucosa intestinal que podem ocasionar efeitos colaterais como: náuseas, vômitos, diarreia, disfagia, estomatites, mucosites, constipação e estufamento abdominal, também agem sobre o sistema imunológico levando a imunossupressão. Devido aos transtornos gastrintestinais muitos pacientes deixam de se alimentar, pois na maioria dos casos apresentam incômodo ao mastigar e deglutir, outros apresentam inapetência ou 


\section{RECIMA21 - REVISTA CIENTÍFICA MULTIDISCIPLINAR ISSN 2675-6218}

CONTRIBUTOS DA ALIMENTAÇÃO SAÚDAVEL COMO ESTRATÉGIA DE PREVENÇÃO E ENFRENTAMENTO DO CÂNCER: UM PERSPECITIVA DA ENFERMAGEM Amanda de Araujo Franco, Amanda de Almeida Floriano, Ana Beatriz Teodoro de Souza, Bianca Lemos de Carvalho, Jully Camara Guinancio, Júlio Gabriel Mendonça de Sousa, Wanderson Alves Ribeiro

saciedade precoce, acarretando em anorexia e desnutrição, podendo evoluir a caquexia, interferindo na qualidade de vida. ${ }^{25,26,27}$

A assistência nutricional ao paciente acometido com câncer de intestino deve ser individualizada e precisa abordar desde a avaliação nutricional, o cálculo das necessidades nutricionais e a terapia nutricional, até o seguimento ambulatorial com o objetivo de prevenir ou reverter o declínio nutricional do paciente. ${ }^{28,29}$ As necessidades nutricionais do organismo e a desnutrição são muito frequentes em consequência da presença do tumor. As alterações metabólicas inerentes ao crescimento dos tumores, como: aumento da lipólise, da utilização de glicose, da produção de marcadores inflamatórios, aumento do gasto energético e inapetência, liberação de substâncias catabólicas etc., além do tratamento antineoplásico, contribuem para a depleção nutricional e a redução da qualidade de vida (QV) do paciente. ${ }^{30}$

Após uma análise no impacto do tratamento no estado nutricional e na QV de pacientes oncológicos, observou-se um baixo consumo de frutas, verduras e legumes. A redução do consumo alimentar pode ser justificada pelas alterações metabólicas e digestivas inerentes ao tratamento antineoplásico, como: inapetência, vômitos, gastroparesia, hipocloridria, náuseas, disfagias, saciedade precoce e má absorção. Silva et al. ${ }^{[31]}$ diz que tais achados se comparam à tendência nacional do baixo consumo desses vegetais em toda a população brasileira, não sendo um achado exclusivo de pacientes neoplásicos. Esse quadro se configura possivelmente pelo aumento do consumo de produtos industrializados, realização de refeições fora de casa, em razão da escassez de tempo em que vivem as famílias brasileiras.

O perfil alimentar observado esteve dentro das recomendações do Guia Alimentar para a População Brasileira ${ }^{21}$, grande parte dos entrevistados retiram a gordura aparente das carnes, usam pouco sal, utilizam óleos vegetais no preparo dos alimentos e realizam todas as refeições diárias. Vale ressaltar que é possível que os entrevistados tenham mudado seu padrão alimentar assim que tomaram ciência do seu estado patológico, sabendo que uma alimentação saudável pode ser benéfica para eles. Salci e Marcon ${ }^{32}$ observaram mudanças nos hábitos alimentares de pacientes e de seus familiares pós-diagnóstico. Eles passaram a ingerir alimentos mais saudáveis e, em alguns casos, as mudanças perduraram após cinco anos de conhecimento da doença.

Oliveira et al. ${ }^{33}$, explicam que, para alguns pacientes os efeitos colaterais do câncer e de seu tratamento influenciam e dificultam a alimentação adequada. Comprovando através de estudos que a dieta é etapa fundamental no tratamento do câncer de intestino, ingerir alimentos adequados antes, durante e após o tratamento auxilia para que o paciente tenha uma melhora significativa e possa se sentir melhor. O consumo de frutas, verduras e legumes pode ser aumentado para que pacientes em tratamento oncológico tenham uma alimentação mais saudável e equilibrada, a fim de acelerar o processo de cura, melhorar o estado nutricional e a adesão ao 


\section{RECIMA21 - REVISTA CIENTÍFICA MULTIDISCIPLINAR ISSN 2675-6218}

CONTRIBUTOS DA ALIMENTAÇÃO SAÚDAVEL COMO ESTRATÉGIA DE PREVENÇÃO E ENFRENTAMENTO DO CÂNCER: UM PERSPECITIVA DA ENFERMAGEM Amanda de Araujo Franco, Amanda de Almeida Floriano, Ana Beatriz Teodoro de Souza, Bianca Lemos de Carvalho, Jully Camara Guinancio, Júlio Gabriel Mendonça de Sousa, Wanderson Alves Ribeiro

tratamento, além de reduzir a morbimortalidade, mudando o perfil do prognóstico desses indivíduos. $^{34}$

\section{CATEGORIA 3 - ESTRATÉGIAS DE ENFRENTAMENTO FRENTE A NECESSIDADE DE MELHORA EM QUALIDADE DE VIDA NO ÂMBITO NUTRICIONAL NA PERSPECTIVA DA ENFERMAGEM}

WCRF e IARC fizeram algumas recomendações para prevenção do câncer, dentre as quais se cita obter e manter um peso corporal saudável, incluir atividades físicas na rotina diária, limitar o consumo de alimentos altamente calóricos, de sal, de bebidas alcoólicas e de carnes vermelhas e processados, evitar bebidas açucaradas, grãos e cereais mofados; também, alerta-se para a necessidade da amamentação tanto para o bebê quanto para a lactante e indicase o consumo de frutas e hortaliças. O Instituto Nacional do Câncer (INCA) considera estas recomendações como sendo de uso individual e sugere ações para organizações multinacionais, organizações da sociedade civil, governo, indústria, mídia, escolas, locais de trabalhos, todos os profissionais e pessoas. ${ }^{35,21,36}$

Preservar o estado nutricional tem efeito direto na qualidade de vida de pacientes com câncer, contribuindo em resultados terapêuticos aumentando expectativa de vida dos pacientes. Entre as diversas estratégias que englobam o tratamento oncológico, tem-se evidenciado na literatura os efeitos positivos da atividade física na qualidade de vida desses pacientes. A atividade física, quando realizada de maneira regular, também vem demonstrando ser um opositor aos efeitos deletérios do tratamento do paciente já doente. ${ }^{27}$

Os hábitos alimentares estão diretamente ligados com a questão do sobrepeso e obesidade entre os adolescentes, que demonstra um grande problema de saúde pública. Nesse contexto o Ministério da Saúde (MS) apresenta uma das políticas públicas para minimizar o problema do excesso de peso em questão. O Programa Saúde na Escola (PSE) é hoje uma das principais políticas públicas para infância e adolescência. Dentre seus componentes evidencia-se a avaliação clínica, nutricional, promoção da alimentação saudável, avaliação oftalmológica e as ações de educação permanente em saúde, atividade física, promoção da cultura da prevenção no âmbito escolar e inclusão das temáticas de educação em saúde no projeto político pedagógico das escolas. ${ }^{37}$

Para Gubert e Prado ${ }^{38}$ a capacitação pedagógica do enfermeiro em sua formação inicial deve estar aliada aos processos de formação permanente das escolas. Espera-se que os educadores preparem profissionais que sejam competentes, críticos, criativos, inovadores, e que possam enfrentar os desafios do mundo do trabalho.

A ação educativa em saúde se refere às atividades voltadas para o desenvolvimento de capacidades individuais e coletivas visando à melhoria da qualidade de vida e saúde. $\mathrm{O}$ profissional de enfermagem deve atuar junto à comunidade, através das associações de bairro, 


\section{RECIMA21 - REVISTA CIENTÍFICA MULTIDISCIPLINAR ISSN 2675-6218}

CONTRIBUTOS DA ALIMENTAÇÃO SAÚDAVEL COMO ESTRATÉGIA DE PREVENÇÃO E ENFRENTAMENTO DO CÂNCER: UM PERSPECITIVA DA ENFERMAGEM Amanda de Araujo Franco, Amanda de Almeida Floriano, Ana Beatriz Teodoro de Souza, Bianca Lemos de Carvalho, Jully Camara Guinancio, Júlio Gabriel Mendonça de Sousa, Wanderson Alves Ribeiro

sindicatos, associações esportivas, igrejas, clubes, entidades culturais, incentivando a participação integrada na busca pela qualidade de vida, servindo de suporte para as mudanças no estilo de vida do indivíduo e da comunidade e contribuindo para o desenvolvimento social. ${ }^{18}$

Verifica-se que, mesmo com todos os alertas apresentados pelos projetos de educação nutricional, ainda é elevado o consumo de alimentos e bebidas processados, os quais se caracterizam por serem ricos em gorduras e açúcares, o que auxilia no aumento dos índices de sobrepeso, de obesidade e de outras doenças crônicas na população. ${ }^{[39]}$ A orientação alimentar é ferramenta de grande utilidade tanto para a promoção de hábitos saudáveis quanto para a prevenção e controle de excesso de peso. ${ }^{40}$ Todos os profissionais devem ter como prioridade a manutenção da saúde pública coletiva, em especial, os profissionais da saúde precisam educar, conscientizar e incentivar a população a ter hábitos saudáveis e a prevenir o câncer. ${ }^{20}$

\section{CONSIDERAÇÕES FINAIS}

Em virtude dos fatos narrados anteriormente, observou-se que a obesidade vem emergindo de forma preocupante no mundo todo. As modificações e atualizações na sociedade contribuíram para mudanças drásticas nos hábitos alimentares e escassez da atividade física. $\mathrm{O}$ aumento do índice de obesidade se relaciona com um risco maior para o desenvolvimento de possíveis tipos de cânceres. Ocorre que, o alto consumo de alimentos processados e/ou industrializados juntamente com uma baixa ingestão de grãos, ocasiona um cenário alimentar maléfico somatizado a um estilo de vida sedentário, sendo apontados como fortes fatores de risco para a instalação de um quadro neoplásico.

Conhecer tais fatores é um estímulo para as mudanças comportamentais de toda a população, visto que a prevenção e controle da obesidade, bem como a melhora no estado nutricional influenciam não somente no bem-estar e na qualidade de vida do ser humano, mas também participam do processo de preservação e promoção da saúde, contribuindo para o equilíbrio do metabolismo, reduzindo os riscos para o surgimento de doenças crônicas. Nesse contexto o papel educativo do enfermeiro se destaca, objetivando a ascendência de uma alimentação e estilo de vida saudável, além da reversão no atual quadro epidemiológico que se encontra a obesidade, consequentemente a morbimortalidade por câncer.

A preservação nutricional também destaca maior efeito na qualidade de vida e no bem-estar de pacientes oncológicos, auxiliando nos resultados dos tratamentos, na evolução prognóstica e aumento da sobrevida. Fundamentando-se nos estudos analisados, fica claro que manter uma dieta equilibrada e a prática de atividades físicas, apontam a prevenção como melhor etiologia para luta contra uma doença de tão grande complexidade e associada à dor física e sentimental.

Concluindo-se que para promover orientações sobre alimentação saudável e nutrição adequada é preciso estar atento ao contexto social cujos indivíduos estão inseridos, abordando 


\section{RECIMA21 - REVISTA CIENTÍFICA MULTIDISCIPLINAR ISSN 2675-6218}

CONTRIBUTOS DA ALIMENTAÇÃO SAÚDAVEL COMO ESTRATÉGIA DE PREVENÇÃO E ENFRENTAMENTO DO CÂNCER: UM PERSPECITIVA DA ENFERMAGEM Amanda de Araujo Franco, Amanda de Almeida Floriano, Ana Beatriz Teodoro de Souza, Bianca Lemos de Carvalho, Jully Camara Guinancio, Júlio Gabriel Mendonça de Sousa, Wanderson Alves Ribeiro

estratégias individualizadas em concordância com a necessidade exposta para uma eficaz manutenção da saúde pública.

\section{REFERÊNCIAS}

1. Dias LZ, et al. A Importância do Ginecologista na Prevenção do Câncer de Mama. Revista de Saúde. Vassouras. 2012;3(1):5-12.

2. Oliveira VA, et al. Relação entre consumo alimentar da população nordestina e o alto índice de câncer gástrico nesta região. Rev. Inter Revista Intertox de Toxicologia, Risco Ambiental e Sociedade. 2014;7(3):06-24.

3. Garófolo A, et al. Dieta e Câncer: Um Enfoque Epidemiológico. Rev. Nutr. Campinas. 2004;17(4):491-505.

4. Informativo da Organização Pan-Americana de Saúde - OPAS/OMS. Brasil. [Acesso em 21 jan. 2021]. Disponível em: https://www.paho.org/pt/topicos/cancer

5. Gurgel MMS. Câncer de Mama: Estágio no Momento do Diagnóstico em Mulheres Residentes do Município Recife-Pernambuco. [Monografia de Especialização em Saúde Coletiva]. Recife: Fundação Oswaldo Cruz. Centro de Pesquisas Aggeu Magalhães; 2011.

6. Friedrich RRA. Influência da Alimentação no Câncer Colo Retal. [Monografia de Especialização em Saúde Pública]. Porto Alegre: Universidade Federal do Rio Grande do Sul; 2008.

7. Prado BBF. Influência dos hábitos de vida no desenvolvimento do câncer. Cienc. Cult. São Paulo. 2014;66(1):21-24.

8. Hipólito KPP, Ribeiro KARA. Importância da Nutrição na Prevenção e no Tratamento de Neoplasias. Interciência \& Sociedade. 2014;3(2):51-59.

9. Farias JF. Prevenção do Câncer Através da Alimentação. III Amostra de Trabalhos de Pós Graduação. XVIII Simpósio de Iniciação Científica. UniFil. Out; 2010.

10. MINISTÉRIO DA SAÚDE, INCA. Ações de Enfermagem para o Controle do Câncer uma Proposta de Integração Ensino-Serviço. $3^{a}$ Edição Revista, Atualizada e Ampliada. Rio de Janeiro; 2016.

11. Organização Pan-Americana de Saúde - OPAS/OMS. Modelo de Perfil Nutricional da Organização Pan-Americana da Saúde. Brasil, 2016. [Acesso em 21 jan. 2021]. Disponível em: https://iris.paho.org/handle/10665.2/18623.

12. IARC. International Agency for Research on Cancer. Monographs evaluate consumption of red meat and processed meat. Lyon:. World Health Organization, Oct; 2015.

13. Gil AC. Métodos e técnicas de pesquisa social. 6. ed. São Paulo: Atlas; 2008.

14. Lakatos EM, Marconi AA. Metodologia científica. 4. ed. São Paulo: Atlas; 2010.

15. Munhoz MP, et al. Efeito do exercício físico e da nutrição na prevenção do câncer. Rev. Odontol. Araçatuba. 2016:34-40. 


\section{RECIMA21 - REVISTA CIENTÍFICA MULTIDISCIPLINAR ISSN 2675-6218}

CONTRIBUTOS DA ALIMENTAÇÃo SAÚDAVEL COMO ESTRATÉGIA DE PREVENÇÃO E ENFRENTAMENTO DO CÂNCER: UM PERSPECITIVA DA ENFERMAGEM Amanda de Araujo Franco, Amanda de Almeida Floriano, Ana Beatriz Teodoro de Souza, Bianca Lemos de Carvalho, Jully Camara Guinancio, Júlio Gabriel Mendonça de Sousa, Wanderson Alves Ribeiro

16. Silva DRQ, Santana AG, Reis AS, Ceolato LO. Mendes RG, Silva S, Cuvello LCF. Relato de experiência: alimentação saudável. São Paulo; 2019;9(4):34-52.

17. Arantes BFR, Martins EM, Alves MA. Obesidade como fator de risco para o câncer: uma nova visão para a enfermagem. Remas-Revista Educação, Meio Ambiente e Saúde; 2019;9(2):63-81.

18. Almeida, $L$ et al. Alimentação como fator de risco para câncer de intestino em universitários. Revista Brasileira em Promoção da Saúde. 2017;30(1).

19. Nascimento, FSM. A importância do acompanhamento nutricional no tratamento e na prevenção do câncer. Caderno de Graduação-Ciências Biológicas e da Saúde-UNIT-SERGIPE. 2015;2(3):11-24.

20. Ministério da Saúde (BR). Política nacional de alimentação e nutrição. Brasília, DF: Ministério da Saúde; 2013.

21. Nix SW. Nutrição e dietoterapia básica. Rio de Janeiro: Elsevier; 2010.

22. Controle do Câncer: uma proposta de integração ensino-serviço. 1993.

23. Salimena AMO, et al. Como Mulheres Submetidas à Quimioterapia Antineoplásic a Percebem a Assistência de Enfermagem. Revista Brasileira de Cancerologia. Rio de Janeiro; 2010;56(3):331-340.

24. Sawada NO, et al. Avaliação da Qualidade de Vida de Pacientes Com Câncer Submetidos à Quimioterapia. Revista da Escola de Enfermagem da USP. São Paulo; 2009;43(3):581-587.

25. Azevedo DC, Bosco SMD. Perfil nutricional, dietético e qualidade de vida de pacientes em tratamento quimioterápico. Universidade Nove de Julho, São Paulo. ConScientiae Saúde. 2011;10(1):23-30.

26. Guimarães AGC, Anjos ACY. Caracterização Sociodemográfica e Avaliação da Qualidade de Vida em Mulheres com Câncer de Mama em Tratamento Quimioterápico Adjuvante. Revista Brasileira de Cancerologia. Rio de Janeiro; 2012;58(4):581-592.

27. Davies M. Nutritional screening and assessment in cancer-associated malnutrition. European Journal of Oncology Nursing. 2015;9:564-573.

28. Marian AE. Nutritional support strategies for malnourished cancer patients. European Journal of Oncology Nursing. 2005;9:74-83.

29. Port GZ. Avaliação nutricional bioquímica de pacientes portadores de cirrose com carcinoma hepatocelular [dissertação]. Porto Alegre: Universidade Federal de Ciências da Saúde de Porto Alegre; 2012.

30. Silva AC, Alves RC, Pinheiro LS. As implicações da caquexia no câncer. E-Scientia. 2012;5(2):49-56.

31. Salci MA, Marcon SS. Enfrentamento do câncer em família. Texto \& Contexto Enfermagem. 2011;20:178-186.

32. Oliveira HSD, et al. Imunonutrição e o tratamento nutricional do câncer. Rev. Ciência \& Saúde, Porto Alegre, 2010;3(2):59-64. 


\section{RECIMA21 - REVISTA CIENTÍFICA MULTIDISCIPLINAR ISSN 2675-6218}

CONTRIBUTOS DA ALIMENTAÇÃO SAÚDAVEL COMO ESTRATÉGIA DE PREVENÇÃO E ENFRENTAMENTO DO CÂNCER: UM PERSPECITIVA DA ENFERMAGEM Amanda de Araujo Franco, Amanda de Almeida Floriano, Ana Beatriz Teodoro de Souza, Bianca Lemos de Carvalho, Jully Camara Guinancio, Júlio Gabriel Mendonça de Sousa, Wanderson Alves Ribeiro

33. Pereira PL, Nunes ALS, Duarte SFP. Qualidade de vida e consumo alimentar de pacientes oncológicos. Revista Brasileira de Cancerologia. 2015;61(3):243-251.

34. World Cancer Research Fund/American Institute for Cancer. Research. Food, nutrition and the prevention of cancer: a global perspective. Washington DC. 1997

35. Mahan LK, Escott-Stump SK. Alimentação, nutrição e dietoterapia. 12. ed. Rio de Janeiro: Elsevier; 2010. p. 1358.

36. Machado MFAS, et al. Programa Saúde na Escola: Estratégia promotora de saúde na atenção básica no Brasil. J. Hum. Growth Dev. São Paulo; 2015;25(3):307-3012.

37. Gubert E, Prado ML do. Desafios na prática pedagógica na educação profissional em enfermagem. Revista Eletrônica de Enfermagem. 2011;13(2):285-95.

38. Pimenta NG, Malhão TA, Gomes FS, Couto SG, Melo MELD. Efeito da atividade educativa "Armazém da Saúde" na promoção da alimentação saudável e prevenção de câncer no ambiente de trabalho. Rev Bras Cancerol. 2015;61(3):205-15.

39. Departamento de Atenção Básica, Secretaria de Atenção à Saúde, Ministério da Saúde. Estratégias para o cuidado da pessoa com doença crônica: obesidade. Brasília: Ministério da Saúde; 2014. (Cadernos de Atenção Básica, 38). 